\title{
ANTS 2010 special issue
}

\section{Editorial}

\section{Marco Dorigo • Mauro Birattari · Gianni Di Caro • René Doursat • Andries Engelbrecht • Luca Maria Gambardella • Roderich Groß • Erol Şahin • Thomas Stüitzle}

Received: 4 November 2011 / Accepted: 5 November 2011 / Published online: 18 November 2011

(C) Springer Science + Business Media, LLC 2011

M. Dorigo $(\bowtie) \cdot$ M. Birattari · T. Stützle

IRIDIA, Université Libre de Bruxelles, Brussels, Belgium

e-mail: mdorigo@ulb.ac.be

M. Birattari

e-mail: mbiro@ulb.ac.be

T. Stützle

e-mail: stuetzle@ulb.ac.be

G. Di Caro · L.M. Gambardella

"Dalle Molle" Institute for Artificial Intelligence Studies (IDSIA), USI-SUPSI, Manno, Switzerland

G. Di Caro

e-mail: gianni@idsia.ch

L.M. Gambardella

e-mail: luca@idsia.ch

R. Doursat

Complex Systems Institute, CREA, Ecole Polytechnique \& CNRS, Paris, France

e-mail: rene.doursat@polytechnique.edu

A. Engelbrecht

CIRG, Department of Computer Science, University of Pretoria, Pretoria, South Africa

e-mail: engel@cs.up.ac.za

R. Groß

Natural Robotics Lab, Department of Automatic Control and Systems Engineering, The University of Sheffield, Sheffield, UK

e-mail: r.gross@sheffield.ac.uk

E. Şahin

KOVAN Research Lab, Department of Computer Engineering, Middle East Technical University,

Ankara, Turkey

e-mail: erol@ceng.metu.edu.tr 
ANTS is the oldest conference series dedicated to the dissemination of swarm intelligence research. Its first edition took place in 1998, at the Université Libre de Bruxelles, Brussels, Belgium. Since then it has been held every other year. This special issue of Swarm Intelligence collects extended versions of some of the best papers published in the proceedings of ANTS 2010, the Seventh International Conference on Swarm Intelligence, which took place in Brussels on September 8-10, 2010.

The authors of the 28 papers accepted as full papers at the conference were invited to submit an extended version of their work for possible inclusion in the special issue. Sixteen authors submitted an extended version and nine of these submissions were accepted for publication after at least one round of reviews with comments by at least three referees. The papers where one of the authors was also one of the editors of this special issue have been managed anonymously by one of the other guest editors.

The special issue is organized in two parts. The first part is this double issue, while the second part will be published as the first issue of year 2012, that is, volume 6 , number 1 .

The special issue opens with "Multiple pheromone types and other extensions to the Ant-Miner classification rule discovery algorithm", by Salama et al. In this paper, the authors propose five possible enhancements of AntMiner, an ant colony optimization-based algorithm for the discovery of classification rules introduced by Parpinelli et al. ${ }^{1}$ The authors compare different combinations of these five enhancements using as a benchmark 23 datasets. They experimentally show that some of these combinations improve the original algorithm performance both in terms of predictive accuracy and of simplicity of the generated rule set.

The second paper is titled "Slime mold inspired routing protocols for wireless sensor networks", by Li et al. In the paper, the authors investigate two self-organizing routing protocols for wireless sensor networks that are inspired by the way slime-molds self-assemble their own resource distribution network. In the paper, the proposed protocols are evaluated via extensive simulations considering both routing and forwarding in networks with single and multiple sinks. Simulation results show that the protocols are effective in establishing network connections with a good balance of efficiency and robustness that is precisely controlled through the model parameters.

The third and fourth papers are about morphogenetic engineering (ME), a research area within swarm intelligence whose goal is to combine the self-organization properties of complex biological systems with the architectural properties of engineered systems. ME investigates new hybrid designs that can provide the best of both worlds towards "self-organized architectures". The main purpose of morphogenetic engineering is to abstract the behavior of multicellular organism development or insects' nest construction into new models of heterogeneous and programmable self-assembly.

The contribution by Yamamoto et al., "Recovery properties of distributed cluster head election using reaction-diffusion", focuses on chemical pattern formation as the basis of cellto-cell signaling and distributed coordination for future ME devices. It investigates the computational capabilities of reaction-diffusion Turing systems, such as the Gierer-Meinhardt or Gray-Scott models. The focus here is on how these models can provide a novel substrate for cluster-head election algorithms. Exploiting the well-known spot formation properties of these chemical systems, single cells can be promoted to local "hubs" by decentralized consensus, while hubs' neighborhoods automatically adjust their shape and size. The au-

\footnotetext{
${ }^{1}$ Parpinelli, R. S., Lopes, H. S., \& Freitas, A. A. (2002). Data mining with an ant colony optimization algorithm. IEEE Transactions on Evolutionary Computation, 6(4), 321-332.
} 
thors propose methods to quantify the stability, speed and post-perturbation self-healing properties of this type of collective behavior. This paper offers a fruitful paradigm of morphogenetic swarm intelligence that can be simulated using cellular automata or by being implemented in spatially scattered processors (e.g., in amorphous computing), or even directly in biochemical matter (e.g., in synthetic biology).

In Beal's paper, "Functional blueprints: an approach to modularity in grown systems", the author investigates how morphogenetic developmental systems are able to maintain functional viability at all stages of their growth, without disruption caused by stress. To address this problem, he proposes to decompose a system $S$ into subsystems $X, Y$, etc., construed as "functional blueprints". These subsystems basically correspond to "viability landscapes" in which the system state's components $C_{X}, C_{Y}$, etc. iteratively move by following a stress gradient and reacting to deviations imposed by the other subsystems. The author then demonstrates the system's tolerance to distortion through elementary geometric examples written in Proto, the spatial programming language that he created. Finally, he presents an application of these principles to the problem of biological tissue growth using an agent-based model of vascularization. By integrating two core properties of grown systems, their viability and modularity, into a single theoretical framework, this study opens the way to promising ME research in artificial development and synthetic biology.

The fifth and sixth papers are about swarm robotics, and conclude Part 1 of the special issue. The first swarm robotics paper, "Task partitioning in swarms of robots: an adaptive method for strategy selection", by Pini et al., studies task partitioning in swarms of robots. Task partitioning is crucial in many situations to reduce interference between workers, to increase energy efficiency and to exploit individual skills. The paper investigates a problem in which a swarm of robots has to decide whether to partition or not a given task into two sub-tasks. The partition decision is made without communication in a self-organized way and is based on individual experiences. Simulation results show that the swarm achieves good performance both when the partition is needed and when it is not needed. The swarm also reacts to on-line changes and the partitioning method scales well to larger swarm sizes.

The second swarm robotics paper, "Majority-rule opinion dynamics with differential latency: a mechanism for self-organized collective decision-making", by Montes de Oca et al., proposes a decision making mechanism that lets a swarm robotics system choose the actions that are shorter in execution time. The mechanism is inspired by models developed in physics that study the dynamics of distributed opinion formation in populations of agents, where each individual changes its opinion to match the one that is accepted by the majority of a subset of the population. The authors extend these models by incorporating latency, a time period of stochastic duration during which the individuals cannot switch opinion. The extended model is first studied analytically, and then validated via an experimental study in which a swarm of simulated mobile robots has to choose between two different routes to a goal location.

Part 2 is dedicated to three papers covering various aspects of ant colony optimization (ACO). The first one, "Theoretical analysis of two ACO approaches for the traveling salesman problem", is a theoretical paper. With this paper, Kötzing et al. contribute to the understanding of theoretical properties of ACO algorithms when applied to NP-hard problems, in particular, the traveling salesman problem (TSP). A first main contribution, which is instrumental for the following results on ACO approximation behavior and runtime bounds, is the analysis of the locality of two construction procedures of ACO algorithms for the TSP. In particular, the authors show that a construction graph that allows ants at each construc- 
tion step to insert an arbitrary edge has a better locality than the classical nearest neighbor based solution construction, which has been used by all the available ACO algorithms for the TSP so far. Using the construction graph with arbitrary edge insertion, the authors then prove new and improved bounds on the expected optimization time of ACO algorithms for a simple TSP instance, which had previously been studied in the ACO literature. They also prove polynomial runtime bounds to reach good approximations for random TSP instances. In addition, the article presents worst case instances for the two ACO construction procedures, for which the expected time to find the optimal solution is exponential in the problem dimension and discusses situations in which a strong influence of the heuristic information is crucial.

The second paper, "A critical analysis of parameter adaptation in ant colony optimization", by Pellegrini et al., reports an empirical study of the performance of five state-of-theart parameter adaptation methods that have been proposed in the literature for ant colony optimization. Parameter adaptation methods operate during the execution of the algorithm, on a per instance basis, to select or constantly modify the parameter setting. In order to study the contribution of the selected parameter adaptation methods independently of the particular algorithms and problems for which they were originally proposed, the authors include the methods in the $\mathcal{M} \mathcal{A} \mathcal{X}-\mathcal{M} \mathcal{I} \mathcal{N}$ Ant System, a well-known ACO algorithm, ${ }^{2}$ and solve a large number of randomly generated instances of the traveling salesman and quadratic assignment problems. The extensive experimental and statistical analyses performed by the authors show that, in the large majority of the cases, the use of the adaptation methods worsens the performance of the $\mathcal{M} \mathcal{A} \mathcal{X}-\mathcal{M I N}$ Ant System.

The third and last paper, "Continuous optimization algorithms for tuning real and integer parameters of swarm intelligence algorithms" by Yuan et al., studies empirically the performance of various continuous optimization algorithms for the offline tuning of numerical parameters in the $\mathcal{M} \mathcal{A} \mathcal{X}-\mathcal{M I N}$ Ant System applied to the traveling salesman problem and in a particle swarm optimization algorithm applied to a family of continuous optimization functions derived from the Rastrigin function. Offline tuning methods select good parameter settings in a preliminary training phase before the actual algorithm is deployed. To study the performance of the continuous optimization algorithms for this task, the authors have defined a set of case studies that differ in the number of parameters to be tuned by each of the two algorithms. In addition, two ways of handling the stochasticity of the algorithms in the offline tuning have been considered: fixed sample size with a novel post-selection through a racing algorithm or the direct application of a racing algorithm. The experiments of the authors show that the two best performing algorithms are CMA-ES and BOBYQA, two state-of-the-art continuous optimization algorithms from the evolutionary and the mathematical programming communities.

The papers published in this special issue are the result of a strong selection: out of the 99 papers submitted to ANTS 2010 only nine papers, less than $10 \%$, found their way into this special issue. Even though this fact alone could explain the high quality of the papers published, the reality is that this excellent result is due to the collective effort of a large number of actors: the authors, who not only submitted very good papers, but who were also willing to revise their papers to accommodate the often substantial requirements made by the referees; the referees, who provided extremely useful comments in a timely manner; and, finally, the many people at Springer who assisted us along the way.

${ }^{2}$ Stützle, T. \& Hoos, H. H. (2000). $\mathcal{M} \mathcal{A} \mathcal{X}-\mathcal{M} \mathcal{I N}$ ant system. Future Generation Computer Systems, 16(8), 889-914. 
So, a great thanks to everybody, and we hope to continue to work with you all in the years to come.

October 2011

\author{
Marco Dorigo \\ Mauro Birattari \\ Gianni Di Caro \\ René Doursat \\ Andries Engelbrecht \\ Luca M. Gambardella \\ Roderich Groß \\ Erol Şahin \\ Thomas Stützle
}

